\title{
Giant impact, planetary merger, and diversity of planetary-core mass
}

\author{
S.-L. Li ${ }^{1,2}$, C. Agnor ${ }^{3}$ and D. N. C. Lin ${ }^{2,4}$ \\ ${ }^{1}$ Dept. of Astronomy, ${ }^{4}$ Kavli Institute of Astronomy \& Astrophysics, \\ Peking University, Beijing 100871, P. R. China \\ email: lisl@vega.pku.edu.cn \\ ${ }^{2}$ Dept. of Astronomy \& Astrophysics, ${ }^{3}$ Dept. of Earth \& Planetary Science, \\ University of California, Santa Cruz, CA 95064, USA
}

\begin{abstract}
Transit observations indicate a large dispersion in the internal structure among the known gas giants. This is a big challenge to the conventional sequential planetary formation scenario because the diversity is inconsistent with the expectation of some well defined critical condition for the onset of gas accretion in this scenario. We suggest that giant impacts may lead to the merger of planets or the accretion of planetary embryos and cause the diversity of the core mass. By using an SPH scheme, we show that direct parabolic collisions generally lead to the total coalescence of impinging gas giants whereas, during glancing collisions, the efficiency of core retention is much larger than that of the envelope. We also examine the adjustment of the gaseous envelope with a 1D Lagrangian hydrodynamic scheme. In the proximity of their host stars, the expansion of the planets' envelopes, shortly after sufficiently catastrophic impacts, can lead to a substantial loss of gas through Roche-lobe overflow. We are going to examine the possibility that the accretion of several Earth-mass objects can significantly enlarge the planets' photosphere and elevate the tidal dissipation rate over the time scale of 100 Myr.
\end{abstract}

\section{Numerical Methods}

We use two methods to simulate giant impacts between gas giants and planetesimals and mergers between two planets. In the first method, we model collisions using smooth particle hydrodynamics (SPH) (see e.g. the reviews by Monaghan (1992)). Its principle virtue for this work is its ability to simulate highly deformed flows and shocks evolving in 3-D. Thus, we are able to calculate the retention efficiency of both protoplanets' core and envelope. However, this method is limited by a small dynamical range in density and short evolution time. One important result from SPH simulations show that spherical symmetry is quickly restored after the giant impact. This allows us to use a 1-D Lagrangian hydrodynamic scheme (OLH) to study the response and evolution of the gaseous envelope after the giant impact. In the 1-D hydrodynamic scheme, we use the EOS from Saumon et al. (1995), and adopt the opacity from Alexander \& Ferguson (1994). We take into account both flux- limited radiation and convective energy transfer in the code. Adaptive mesh is also included.

\section{Results from SPH simulations}

In table 1, we list the parameters and results for the models calculated using SPH scheme. The columns indicate the number of the model, initial total planetary mass of the target, mass of condensed material of the target, mass of the impactor, mass of 
Table 1. Parameters and impact results from SPH simulations

\begin{tabular}{ccccccccc}
\hline Model & $\begin{array}{c}M_{T} \\
\left(M_{\oplus}\right)\end{array}$ & $\begin{array}{c}M_{T, c} \\
\left(M_{\oplus}\right)\end{array}$ & $\begin{array}{c}M_{I} \\
\left(M_{\oplus}\right)\end{array}$ & $\begin{array}{c}M_{I, c} \\
\left(M_{\oplus}\right)\end{array}$ & $\begin{array}{c}\xi \\
\left({ }^{\circ}\right)\end{array}$ & $v_{i m p} / v_{\text {esc }}$ & $\begin{array}{c}M_{f, c} \\
\left(M_{\oplus}\right)\end{array}$ & $\begin{array}{c}M_{f, g} \\
\left(M_{\oplus}\right)\end{array}$ \\
\hline 1 & 100 & $50 \mathrm{fe}$ & 100 & $50 \mathrm{fe}$ & $0^{\circ}$ & 1.0 & 99.7 & 97.3 \\
2 & 100 & $50 \mathrm{fe}$ & 100 & $50 \mathrm{fe}$ & $45^{\circ}$ & 1.0 & 100 & 100 \\
3 & 100 & $50 \mathrm{fe}$ & 100 & $50 \mathrm{fe}$ & $0^{\circ}$ & 1.4 & 98.6 & 60.4 \\
4 & 100 & $50 \mathrm{fe}$ & 100 & $50 \mathrm{fe}$ & $45^{\circ}$ & 1.4 & 49.4 & 35.6 \\
5 & 100 & $50 \mathrm{fe}$ & 25 & $25 \mathrm{~b}$ & $0^{\circ}$ & 1.0 & 75.0 & 50.0 \\
6 & 100 & $50 \mathrm{fe}$ & 25 & $25 \mathrm{~b}$ & $30^{\circ}$ & 1.0 & 74.9 & 49.1 \\
7 & 100 & $50 \mathrm{fe}$ & 25 & $25 \mathrm{~b}$ & $0^{\circ}$ & 1.4 & 74.8 & 46.2 \\
8 & 100 & $50 \mathrm{fe}$ & 25 & $25 \mathrm{~b}$ & $30^{\circ}$ & 1.4 & 52.4 & 47.6 \\
9 & 100 & $10 \mathrm{fe}$ & 10 & $10 \mathrm{~b}$ & $30^{\circ}$ & 1.4 & 10 & 95 \\
\hline
\end{tabular}

condensed material of the impactor, impacting angle, the ratio of impacting velocity to escape velocity, final mass of condensed material and gaseous envelope mass of the planet after the impact. For the composition of condensed material of all the models, 'fe' denotes iron and ' $b$ ' denotes basalt. The first 4 models are simulating the impact between two giant planets. While model 5-9 are simulating the collisions between giant planets and planetesimals. We show that low-velocity collisions result in the merger of the giant planets and the impactors. Also, small impact angles generally lead to the total coalescence of impinging gas giants. While during high-velocity glancing collisions, there is high probability of core erosion and gaseous envelope loss, and the efficiency of core retention is much larger than that of the envelope.

\section{Results from OLH calculations}

We construct the impacted models based on static initial models with various core and envelope masses. Since we are interested in close-in planets, the tidal effect of their host stars are taken into account. We listed the model parameters and results in Table 2. Columns in Table 2 have the similar meanings as in Table 1 . We also include, in the last column, the photospheric radii of the planets immediately after they have reestablished a state of hydrostatic equilibrium following the post-impact envelope expansion. This results show the extent of expansion of the gaseous envelope due to the giant impact. Two series of initial models are considered: Saturn-like models (1013) with $M_{T}=100 M_{\oplus}$ and $M_{T, c}=10 M_{\oplus}$ and HD149026b-like models (14-17) with $M_{T}=110 M_{\oplus}$ and $M_{T, c}=73 M_{\oplus}$. The initial radii of the models with masses of $100 M_{\oplus}$ and $110 M_{\oplus}$ are around $1 R_{J}$ and $0.75 R_{J}$ respectively. With adequate impact energy, we find, in Saturn-like model 13, that giant impacts can significantly enlarge the planets' photosphere. During catastrophic impact (HD149026b-like model 17), the gaseous envelope can be substantially lost as the expansion of the planets' photosphere has largely exceeded its Hill Radius. Even though some of the Saturn-like models are collided by impactor with masses several times larger than that in the catastrophic HD149026blike model 17, there is no significant mass loss in these models. This dichotomy may be accounted for by the following reasons. In the calculations, we assume that all the impactors can reach the core, with ablated mass and gravitational energy deposited in a region around the core. Thus, the deposited energy by the impactor is not only function of the mass of the impactor, but also related to the depositing location and the planetary mass inside the location. For the same amount of material deposited at the same location, the deposited energy would be larger in the HD149026b-like models than that in the Saturn-like models, because the former have much larger solid core than the latter. 
Table 2. Parameters and impact results from OLH simulations

\begin{tabular}{ccccccccc}
\hline Model & $\begin{array}{c}M_{T} \\
\left(M_{\oplus}\right)\end{array}$ & $\begin{array}{c}M_{T, c} \\
\left(M_{\oplus}\right)\end{array}$ & $\begin{array}{c}M_{I} \\
\left(M_{\oplus}\right)\end{array}$ & $M_{I, c}$ & $\begin{array}{c}v_{i m p} / v_{\text {esc }} \\
\left(M_{\oplus}\right)\end{array}$ & $\begin{array}{c}M_{f, c} \\
\left(M_{\oplus}\right)\end{array}$ & $M_{f, g}$ & $R_{\tau} / R_{J}$ \\
\hline 10 & 100 & 10 & 10 & 10 & 1.0 & 10 & 100 & 1.13 \\
11 & 100 & 10 & 50 & 50 & 1.0 & 10 & 140 & 1.40 \\
12 & 100 & 10 & 50 & 50 & 1.0 & 60 & 90 & 1.50 \\
13 & 100 & 10 & 100 & 10 & 1.0 & 20 & 180 & 1.62 \\
14 & 110 & 73 & 15 & 15 & 1.0 & 73 & 52 & 1.06 \\
15 & 110 & 73 & 15 & 15 & 1.0 & 88 & 37 & 1.03 \\
16 & 110 & 73 & 7.5 & 7.5 & 1.4 & 73 & 44.5 & 1.03 \\
17 & 110 & 73 & 37 & 37 & 1.0 & 73 & - & - \\
\hline
\end{tabular}

And a side effect of the massive impactors is that the Hill radius will increase with the increasing mass of impactor, which will make it more difficult for the envelope to expand out of its Hill radius. Furthermore, the Saturn-like models have very massive gaseous envelope compared with the HD149026b-like models. They obviously require much larger energy deposition to overcome the gravitational binding for the envelope to escape out of the Hill radius. In the future work, we want to examine the long-term evolution of the impacted models, in order to explore the possibility that the expansion of the planets after the impact can last for over an observable timescale.

\section{References}

Saumon, D., Chabrier, G., \& van Horn, H. M. 1995, ApJS, 99, 713

Alexander, D. R. \& Ferguson, J. W. 1994, ApJ, 437, 879

Monaghan, J. J. 1992 ARAA, 30, 543 